\title{
COMUNICAÇÃO CIENTÍFICA \\ Variações morfológicas em Olindias sambaquiensis (Cnidaria, Hydrozoa, Limnomedusae) no litoral de Guaratuba, Paraná, Brasil ${ }^{1}$
}

\author{
Miodeli Nogueira Júnior ${ }^{2} \&$ Maria A. Haddad ${ }^{2}$ \\ 1 Contribuição número 1622 do Departamento de Zoologia, Universidade Federal do Paraná. \\ ${ }^{2}$ Departamento de Zoologia, Universidade Federal do Paraná. Caixa Postal 19020, 81531-980 Curitiba, Paraná, Brasil. \\ E-mail:miodeli@gmail.com; mahaddad@ufpr.br
}

\begin{abstract}
Morphological variations in Olindias sambaquiensis (Cnidaria, Hydrozoa, Limnomedusae) in the coast of Guaratuba, Paraná, Brazil. Olindias sambaquiensis Muller, 1861 (Olindiidae) is an abundant and common hydromedusae in the southern Brazilian littoral region. Morphology of 1092 specimens collected in coastal waters at Guaratuba, in Paraná, southern Brazil, was studied. The form, number and branching of the radial and centripetal canals were variable in only 70 individuals $(6.4 \%)$, which, in some cases, were associated with apparent changes in symmetry pattern.
\end{abstract}

KEY WORDS. Hydromedusae; morphology.

RESUMO. Olindias sambaquiensis Muller, 1861 (Olindiidae) é uma hidromedusa comum e abundante no litoral Sul do Brasil. Em contribuição ao estudo da espécie, variações morfológicas foram analisadas em 1092 exemplares coletados em águas costeiras de Guaratuba, Paraná. Em 70 (6,4\%) indivíduos, foram reconhecidas alterações relacionadas ao número, forma e ramificações dos canais radiais e centrípetos, que resultaram, em diversos casos, em uma aparente mudança do padrão de simetria da espécie.

PALAVRAS-CHAVE. Hidromedusa; morfologia.

\begin{abstract}
Variações morfológicas em hidromedusas são freqüentes e têm sido descritas desde as últimas décadas do século XIX. HargitT (1901), em um dos trabalhos mais completos sobre o assunto, discute diversos trabalhos anteriores e relata a variabilidade em sete espécies, destacando-se o estudo minucioso sobre variações em mais de $15 \%$ dos exemplares de uma espécie não identificada de Gonionemus A. Agassiz, 1862. Alterações morfológicas marcantes, tais como a poligastria (dois ou mais estômagos separados) e as medusas duplas ou siamesas, são raras. As variações mais comuns são de natureza merística, relacionadas principalmente ao número de canais radiais, que implicam em diferenças de número de gônadas e tentáculos marginais (Hargitt 1901, Russell 1953, Navas-Pereira 1984, Zamponi \& Genzano 1989a, SiLva et al. 2003).

De acordo com Russell (1953), a freqüência de exemplares alterados geralmente é baixa, mas pode variar bastante de acordo com a espécie e a população estudada: 5-6\% em Obelia sp. e 6\% em Blackfordia virginica Mayer, 1910 (Silva et al. 2003); 8\% em Moerisia lyonsi Boulenger, 1908 (Boulenger 1908). O mesmo autor, entretanto, cita algum tipo de variação estrutural em mais de 35\% dos indivíduos de Eirene Eschscholtz, 1829 e de Helgicirrha Hartlaub, 1909. NAVAs-Pereira (1984) encontrou
\end{abstract}

um número de canais radiais diferente de quatro em 90\% dos indivíduos de Phialucium carolinae (Mayer 1900) do Indo-Pacífico, mas nenhuma variação em populações do Sul do Brasil.

Olindias sambaquiensis Muller, 1861 (Limnomedusae, Olindiidae) é endêmica do Atlântico Sudoeste e distribui-se entre São Paulo e Mar del Plata (Vannucci 1951, Mianzan 1989), onde é comum e abundante (VANNUCCI 1951, ZAMPONI \& FACAL 1987, MiANZAN \& ZAMPONI 1988), além de freqüentemente estar associada a queimaduras em banhistas e pescadores (VANNUCCI 1951, Zamponi \& Facal 1987, Haddad Jr et al. 2002). A morfologia de indivíduos adultos (VANNUCCI 1951) e o desenvolvimento da fase medusóide, de juvenil até adulto (ZAMPONI \& Girola 1989), já foram descritos em detalhes e sua diminuta fase polipóide já foi obtida em laboratório (Zamponi \& FACAL 1987). Variações morfológicas, porém, não foram ainda tratadas nesta espécie, exceto na breve citação de ZAMPONI \& Girola (1989) sobre exemplares jovens com cinco canais radiais. Quando modificações do número de estruturas e da simetria levam a descrições de novos taxa, podem surgir questionamentos taxonômicos, a exemplo dos novos gêneros de Geryonidae descritos por ZAMPONI \& GENZANO (1989a,b), que foram questionados por BoullLon (1999) e posteriormente aceitos por BouILLON \& BOERo (2000).

Revista Brasileira de Zoologia 23 (3): 879-882, setembro 2006 
Assim, o conhecimento dos possíveis "desvios" estruturais a que uma espécie ou população está predisposta, pode esclarecer as dúvidas taxonômicas relacionadas à identidade de alguns espécimes variantes. Este trabalho contribui, portanto, com a descrição e análise morfológica de O. sambaquiensis e também com a taxonomia da Família Olindiidae, caracterizando as alterações estruturais encontradas em exemplares coletados em águas costeiras de Guaratuba, Paraná.

Coletas mensais foram realizadas no litoral de Guaratuba, Paraná (entre $25^{\circ} 20^{\prime}-25^{\circ} 55^{\prime} \mathrm{S}, 48^{\circ} 10^{\prime}-48^{\circ} 35^{\prime} \mathrm{W}$ ), entre fevereiro de 2003 e dezembro de 2004, entre 8 e $14 \mathrm{~m}$ de profundidade, utilizando-se arrastos de fundo e de superfície, com redes de malha de 2 e $1 \mathrm{~cm}$ de abertura. Os animais foram anestesiados com cristais de mentol e posteriormente fixados em solução de formol salino a 4\%. Cada exemplar de O. sambaquiensis teve seu diâmetro umbrelar medido após a fixação e sua morfologia foi comparada a descrições prévias (VANnUCCI 1951). Foram considerados como "variantes" os indivíduos que apresentavam uma ou mais características diferentes daquelas descritas para a espécie, conforme o padrão seguinte: a) quatro canais radiais retos, eqüidistantes e não ramificados; b) diversos canais centrípetos retos e não ramificados; c) quatro gônadas papiliformes estendendo-se sobre os canais radiais (Fig. 1). Dez exemplares de referência foram depositados no Museu de Zoologia da Universidade de São Paulo sob o número MZUSP-900.

Foram analisados 1092 indivíduos de O. sambaquiensis, entre 7-80 mm de diâmetro. Do total, 1022 apresentaram características consideradas normais para a espécie e $70(6,4 \%)$, alguma alteração morfológica relacionada aos canais radiais e centrípetos. As variações foram agrupadas em 4 tipos (Figs 216): 1) variação no número de canais radiais (entre 2 e 5 ; 40 exemplares); 2) mudança de simetria dos canais radiais (37 exemplares); 3) ramificação de canais (radiais, centrípetos ou ambos; 34 exemplares); 4) ligação lateral entre canais (radiais, centrípetos ou ambos; 25 exemplares) em diversos graus, alguns com tendência a anastomose. Foram encontrados diversos exemplares com características de dois ou mais dos "grupos" apresentados acima, como por exemplo, um exemplar com três canais radiais, um destes ramificado, e ainda com canais centrípetos unidos (Fig. 3).

As alterações morfológicas relativas ao número, posição e simetria dos canais radiais, conforme as encontradas em $O$. sambaquiensis, também são as mais comuns em outras hidromedusas (Russell 1953). HaRgITT (1901) encontrou alterações nos canais radiais de várias hidromedusas e descreveu detalhadamente as que observou em Gonionemus sp. Nesta espécie, tipicamente com quatro canais radiais, observou variações entre dois e seis em $5 \%$ dos exemplares e alterações de forma, disposição e confluência dos canais em 30\%. Em P. carolinae e $B$. virginica, também com quatro canais radiais, houve variações numéricas entre três a treze na primeira (NAvas-Pereira 1984) e de três, quatro, cinco ou mais na última, além de ramificações, uniões e outras alterações relativas à forma dos canais e à sime- tria (SILVA et al. 2003). O aparecimento de canais anastomosados em alguns exemplares de O. sambaquiensis não é comum em outras hidromedusas, possivelmente devido à presença, proximidade e elevado número dos canais centrípetos em Olindias, que facilitam o surgimento desse tipo de alteração.

A freqüência de exemplares "variantes" geralmente é baixa (Russell 1953), concordando com os resultados obtidos no presente estudo $(6,4 \%)$, embora varie bastante de acordo com a espécie e a população estudada (NAVAs-Pereira 1984).

Variações morfológicas são comuns em medusas, principalmente de simetria, mas não é claro se as causas são genéticas, ambientais, de desenvolvimento, ou de combinações desses fatores (GeRshwin 1999). Também as circunstâncias em que ocorrem alterações em hidromedusas têm sido pouco discutidas na literatura. Somente Russell (1953) aponta para um possível "resultado de condições desfavoráveis, embora não faltem evidências de que, em alguns momentos, possam ser devido a mutações". O mesmo autor comenta que alterações como a poligastria seja um prelúdio à reprodução assexuada. Se assim acontece, outras variações também podem representar respostas ou conseqüências de funções biológicas ainda pouco compreendidas (Silva et al. 2003).

\section{AGRADECIMENTOS}

À CAPES (bolsa de Mestrado) e a dois consultores anônimos.

\section{REFERÊNCIAS BIBLIOGRÁFICAS}

BoullLon, J. 1999. Hydromedusae, p. 385-465. In: D. Boltovskoy (Ed.) South Atlantic Zooplankton. Leiden, Backhuys Publishers, vol. 1, 1500p.

Boulllon, J.\& F. Boero 2000. Synopsis of the families and genera of the Hydromedusae of the world, with a list of the worldwide species. Thalassia Salentina, Nápoles, 24: 47-296.

Boulenger, C.L. 1908. On Moerisia lyonsi a new Hydromedusa from Lake Qurun. Quarterly Journal of Microscopic Science, Londres, 52: 357-378.

Gershwin, L. 1999. Clonal and population variation in jellyfish symmetry. Journal of Marine Biological Association of United Kingdom, Plymouth, 79: 993-1000.

Haddad Jr., V.; F.L. Silveira; J.L.C. Cardoso \& A.C. Morandini. 2002. A Report of 49 cases of cnidarian envenoming from southeastern Brazilian coastal waters. Toxicon, Londres, 40: 1445-1450.

HARGITT, C.W. 1901. Variation among hydromedusae. Biological Bulletin, Woods Hole, 2 (5): 221-255.

Mianzan, H.W. 1989. Distribuición de Olindias sambaquiensis Müller, 1861 (Hydrozoa, Limnomedusae) en el Atlântico Sudocidental. Iheringia, Série Zoologia, Porto Alegre, 69: 155-157.

Mianzan, H.W. \& M.O. Zamponi. 1988. Estudio bioecológico de Olindias sambaquiensis Müller, 1861 (Limnomedusae, Olindi- 


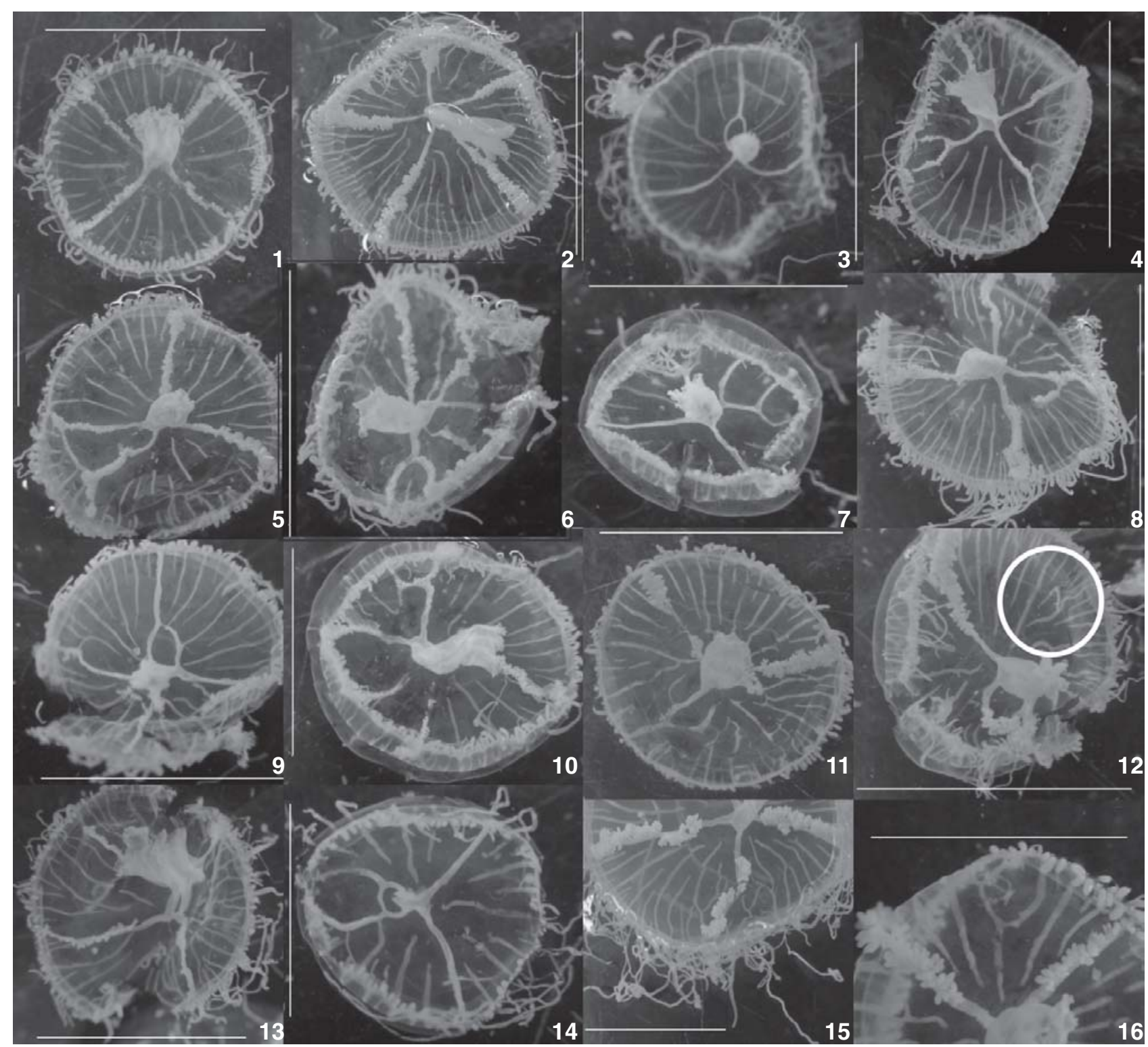

Figuras 1-16. Variações morfológicas encontradas em Olindias sambaquiensis, comparando com o indivíduo padrão. (1) Indivíduo normal, escala = 3,5 cm; (2) com cinco canais radiais eqüidistantes, escala $=3,5 \mathrm{~cm}$; (3) com três canais radiais, um deles ramificado e novamente unido, e alguns canais centrípetos unidos, escala $=2,0 \mathrm{~cm} ;(4)$ com quatro canais radiais eqüidistantes um deles ramificado, escala $=2,5 \mathrm{~cm}$; (5) com quatro canais radiais não eqüidistantes, dois deles se unidos lateralmente interrompendo os canais centrípetos no quadrante, escala $=1,5 \mathrm{~cm}$; (6) três canais radiais não eqüidistantes, dois deles ramificados, escala $=2,5 \mathrm{~cm}$; (7) quatro canais radiais eqüidistantes, um deles divide-se duas vezes, escala $=2,5 \mathrm{~cm}$; (8) quatro canais radiais eqüidistantes, um deles com uma pequena ramificação lateral, escala $=4,0 \mathrm{~cm}$; (9) quatro canais radiais eqüidistantes, dois deles ligados por um prolongamento lateral, canais centrípeto se unem ao prolongamento, escala $=2,3 \mathrm{~cm}$; (10) cinco canais radiais não eqüidistantes, um deles ramificado, escala $=3,0$ $\mathrm{cm}$; (11) três canais radiais eqüidistantes, um deles ramificado e reduzido, escala $=3,5 \mathrm{~cm} ;(12)$ dois canais radiais não equidistantes, canais centrípetos ramificados (círculo), escala $=3,0 \mathrm{~cm} ;(13)$ três canais radiais não eqüidistantes, dois deles quase fundidos próximo a base do manúbrio, escala $=2,3 \mathrm{~cm} ;(14)$ quatro canais radiais não eqüidistantes, dois deles unidos lateralmente, escala $=2,0 \mathrm{~cm} ;(15)$ detalhe de dois quadrantes com canais centrípetos se unindo, escala $=2,0 \mathrm{~cm}$; (16) detalhe de um quadrante com canais centrípetos se unindo, escala = 1,2 cm. Os exemplares das fotos números 7, 8, 9, 12 e 13 tiveram a margem da umbrela cortada e o número 14 o manúbrio totalmente removido, para facilitar a visualização do padrão dos canais.

Revista Brasileira de Zoologia 23 (3): 879-882, setembro 2006 
idae) en el área del Monte Hermoso. II. Factores meteorológicos que influyen su aparicion. Iheringia, Série Miscelânea, Porto Alegre, 2: 63-68.

Navas-Pereira, D. 1984. On the morphological variation of Phialucium carolinae (Mayer, 1900) (Leptomedusae, Phialuciidae). Dusenia, Curitiba, 14 (2): 51-53.

RusSELL, F.S. 1953. The Medusae of British Isles. Hydromedusae. Cambridge University, vol. 1, 530p.

Silva, C.A.; S. Gómez-Aguirre \& G.M. Arce. 2003. Variaciones morfológicas em Blackfordia virginica (Hydroidomedusae: Blackfordiidae) em lagunas costeras de Chiapas, México. Revista de Biologia Tropical, San Jose, 51 (2): 409-412.

VANNUCCI, M. 1951. Hydrozoa e Scyphozoa existentes no Instituto Paulista de Oceanografia I. Boletim do Instituto Oceanográfico da Universidade de São Paulo, São Paulo, 2 (1): 67-98.
Zamponi, M.O. \& C.V. Girola. 1989. Variaciones morfológicas y estructurales de los juveniles de Olindias sambaquiensis Müller, 1861 (Cnidaria, Limnomedusae; Olindiidae). Iheringia, Série Zoologia, Porto Alegre, 69: 19-30.

Zamponi, M.O. \& G.N. Genzano. 1989a. Variaciones de algunas estructuras de valor taxonómico en la Familia Geryonidae (Cnidaria; Trachymedusae) y su relación con la temperatura y salinidad. Iheringia, Série Zoologia, Porto Alegre, 69: 31-47.

Zamponi, M.O. \& G.N. Genzano. 1989b. Nuevas adiciones a la medusofauna de la region subantartica. II. Trachymedusae (Coelenterata: Hydrozoa). Neotropica, La Plata, 34 (91): 3339.

Zamponi, M.O. \& O.N. FaCAL. 1987. Estudio bioecológico de Olindias sambaquiensis Müller, 1861 (Limnomedusae, Olindiidae) en el área del Monte Hermoso. I. Ciclo de vida. Neotropica, La Plata, 33 (90): 109-118.

Recebido em 24.VIII.2005; aceito em 07.VIII.2006.

Revista Brasileira de Zoologia 23 (3): 879-882, setembro 2006 